\title{
Effects of methylprednisolone and treadmill training on spinal cord injury in experimental rats
}

\author{
JIAN-TAO LIU ${ }^{1}$, SHUO-YU WANG ${ }^{1}$, HAN-PING XIAO ${ }^{1}$, BING GU $^{1}$ and HUA-NAN LI ${ }^{2}$ \\ ${ }^{1}$ School of Life Sciences, Jiangxi Science and Technology Normal University, Nanchang, Jiangxi 330013; \\ ${ }^{2}$ Department of Spine Surgery, The Affiliated Hospital of Jiangxi University of \\ Traditional Chinese Medicine, Nanchang, Jiangxi 330006, P.R. China
}

Received March 20, 2020; Accepted July 1, 2021

DOI: $10.3892 / \mathrm{etm} .2021 .10849$

\begin{abstract}
Methylprednisolone (MP) is widely used to treat clinical spinal cord injury (SCI). Treadmill training is also considered an important treatment after SCI to improve motor function in patients, resulting in an evident improvement. Therefore, the present study was designed to evaluate and contrast the effects of MP and treadmill training administered in combination or alone after SCI in adult rats. A rat spinal cord T10 contusion model was induced in Sprague-Dawley rats using an impact device. A total of 40 rats were divided into four groups ( $\mathrm{n}=10$ rats/group): the $\mathrm{MP}, \mathrm{MP}+$ treadmill training, SCI and sham group. At $30 \mathrm{~min}$ after injury, MP sodium succinate was injected into the rats of the MP and $\mathrm{MP}+$ treadmill training groups. Treadmill training began on the second week post-trauma and was performed for 8 weeks. The results showed that MP therapy combined with treadmill training significantly ameliorated several parameters of hind limb function compared with those by MP treatment alone (all $\mathrm{P}<0.05)$. A significantly reduced immunopositive area of Nogo receptor and chondroitin sulfate proteoglycans and reduced relative expression of these mRNAs were found in the $\mathrm{MP}+$ treadmill training group $(\mathrm{P}<0.05)$ compared with the findings in the MP group. In conclusion, the present study indicated that combined MP and treadmill training treatment improved the recovery of hind limb function in rats with SCI, thus potentially representing a promising strategy to cure SCI.
\end{abstract}

\section{Introduction}

Annually, >250,000 individuals worldwide suffer from spinal cord injury (SCI) (1). SCI is an injury to the spinal cord resulting in loss of neurological function. The damage can be divided

Correspondence to: Dr Bing Gu, School of Life Sciences, Jiangxi Science and Technology Normal University, 605 Fenglin Avenue, Nanchang, Jiangxi 330013, P.R. China

E-mail: bguemory@hotmail.com

Key words: methylprednisolone, treadmill training, spinal cord injury, gait analysis, combined therapy into primary and secondary damage (2). Primary damage includes disruption of tissue integrity, blood vessel and axon injuries due to a traumatic event (3). After the initial lesion, several pathophysiological events occur within hours, and include a series of motor, sensory and autonomic dysfunctions with degenerative characteristics (4). Significant advances in pharmacotherapy have been achieved to cease or slow down the secondary damage in SCI (4). To date, methylprednisolone (MP) is the first-line therapeutic option, administrated in the first hours after injury with elevated dosage to reduce severe edema development (5). MP is a unique therapeutic agent validated by the Food and Drug Administration as a standard therapeutic tool in acute SCI management (2). As indicated in the clinical treatment guidelines of SCI, acute SCI should be treated with MP first (5). However, high doses of MP fail to improve neurological recovery if administration of MP is given $8 \mathrm{~h}$ or more after the injury (6). In addition, the continuous use of MP is associated with increased side effects, such as pulmonary emboli, wound infections and severe pneumonia, and it has also failed to improve motor function (7). The steps in late-stage treatment of SCI aim to reduce scar formation, slow down the apoptosis of the remaining neurons and strengthen limb activities (2). Therefore, the combination of MP with other therapeutic approaches that enhance functional recovery, thus reducing the duration and/or dose of the MP treatment, has been extensively studied (8-10).

SCI often results in severe and permanent locomotion deficits. Improvements in medical care after SCI help patients to reduce the effects of injuries, such as deficits in movement, sensitivity and autonomy (11). Treadmill training is one of the interventions aimed at promoting neuroplasticity, axonal sprouting and synapses, and decreasing muscular atrophy following SCI $(12,13)$. Several studies in rodents with complete spinal transection showed that treadmill training significantly improves nerve motor function recovery (13-15) while maintaining axonal activity after SCI through continuous exercise, improves regeneration of spinal cord axons and contributes to neuronal survival (16).

In the late stage of SCI, the secretion of proteoglycan and myelin components accompanied by glial cell hyperplasiainhibit the extension of axons (17). Myelin-associated inhibitory factor is one of the key inhibitory factors of axonal regeneration (2). Nogo receptor $(\mathrm{NgR})$ is a common receptor for three major 
myelin inhibitory proteins, namely Nogo-A, myelin-associated glycoprotein and oligodendrocyte-myelin glycoprotein (18). $\mathrm{NgR}$ expression is upregulated in tissues involved in SCI (18). $\mathrm{NgR}$ directly inhibits axonal regeneration and activation of the associated signaling pathway in the injured area, which are some of the important causes of neurological aplastic disorder after SCI (19). In addition, other factors secreted by astrocytes, such as chondroitin sulfate proteoglycans (CSPGs), contribute to the inhibition of axonal growth (20). Only low levels of CSPGs are expressed in the normal spinal cord, but after SCI, their expression levels increased (20). CSPGs accumulate in the central area of the injury, creating a barrier between the hypertrophic scar tissue and normal tissue (21). With their large size and dense negative charge, CSPGs form a barrier against the growth of new axons in the injured site (19). Indeed, the inhibition of CSPGs expression effectively improves the microenvironment after SCI (22).

At present, there is no systematic approach to restore functions in patients with SCI $(1,2)$. The appropriate treatment and its timing for SCI is yet to be elucidated. Although previous studies have been conducted on MP (5) and treadmill training separately (23), to the best of our knowledge, no studies have reported the effects of combined MP and treadmill training treatment. It is not known whether this combined treatment has synergistic beneficial effects. Therefore, in the present study, rats with SCI were subjected to regular exercise rehabilitation training after MP treatment. Changes in behavior and morphology after SCI were quantitatively observed and evaluated using the TreadScan gait analysis system and professional image analysis system in order to investigate the effects of treadmill training combined with MP therapy on glial scar formation and motor function recovery after SCI, which could potentially represent a promising strategy to cure SCI.

\section{Materials and methods}

Experimental animals. A total of 50 adult (8-week-old) Sprague-Dawley male rats (230-250 g) were obtained from Hunan SJA Laboratory Animal Co., Ltd. (animal certificate no. 43004700000040). The animals were individually housed under a $12 / 12-\mathrm{h}$ light/dark cycle at $22 \pm 2{ }^{\circ} \mathrm{C}$ with $55-65 \%$ humidity and fed with standard chow and water ad libitum. The rats were randomly allocated into a sham operation group (Sham), model group (SCI), treadmill training treatment group (TT), MP treatment group (MP) and MP + treadmill training treatment group ( $\mathrm{MP}+\mathrm{TT})(\mathrm{n}=10$ rats in each group). One week before the SCI surgical procedure, an adaptive training was performed on a computer-controlled animal experiment platform (ZH-PT computer control experimental animal treadmill; Anhui Zhenghua Biologic Apparatus Facilities) in order for the rats to familiarize with the track environment to ensure a continuous run. Every morning and evening, each group was trained for 10 min using a treadmill speed of $20 \mathrm{~m} / \mathrm{min}$. All animal procedures strictly followed the Guide for the Care and Use of Laboratory Animals (23) and were approved by the Jiangxi University of Traditional Chinese Medicine Committee on Animal Research (approval no. JZLLSC2019-0147).

SCI animal model. The SCI animal model was constructed as previously described, with several modifications (24).
Briefly, rats were anesthetized with 5\% chloral hydrate [300 mg/kg, intraperitoneal injection (i.p.); Shanghai yuanye Bio-Technology Co., Ltd.]. Using the T10 spinous process as the center, a $4.0 \mathrm{~cm}$-longitudinal incision was performed to separate the muscles and fascia from both sides of the spine in order to expose the spinal dura mater. A T10 SCI was achieved by the impact of PinPoint ${ }^{\mathrm{TM}}$ Precision Cortex Striker (diameter, $3.0 \mathrm{~mm}$; Hatteras Instruments Inc.). The impact depth was $1.0 \mathrm{~mm}$; the impact velocity was $2.0 \mathrm{~m} / \mathrm{sec}$ and the residence time was $200 \mathrm{msec}$. The sham operation group was subjected to the same procedure described above, but without impact. Post-surgical anti-inflammatory therapy was provided by an intramuscular injection of penicillin and gentamicin sulfate $(25 \mathrm{mg} / \mathrm{kg}$ every $12 \mathrm{~h}$ for 7 days). All rats were administered a daily analgesic, meloxicam $(1 \mathrm{mg} / \mathrm{kg}$, i.p.; Sigma-Aldrich; Merck KGaA), for the first 7 days. In case of urinary tract infection, the anti-inflammatory therapy was prolonged to 10 days. The rectal temperature was maintained at $37 \pm 1^{\circ} \mathrm{C}$ using a thermostatic pad. The injured rats' bladders were manually emptied twice daily until spontaneous voiding occurred.

MP administration and treadmill training. At $30 \mathrm{~min}$ after $\mathrm{SCI}$, rats in the MP and MP + TT group received a bolus injection of MP sodium succinate $(30 \mathrm{mg} / \mathrm{kg}$; Solu-Medrol; cat no. S02340; Pfizer, Inc.) in $0.9 \%$ saline $(0.5 \mathrm{ml})$ via tail vein injection. The rats in the Sham, TT and SCI groups were treated with an injection of $0.5 \mathrm{ml}$ normal saline. A six-lane treadmill (ZH-PT computer control experimental animal treadmill; Anhui Zhenghua Biologic Apparatus Facilities) was used to train the animals and to obtain kinematic data. At 1 week before the SCI surgical procedure, an adaptive training was performed on the aforementioned six-lane treadmill for 6 days with a $10-\mathrm{min}$ running period at $20 \mathrm{~m} / \mathrm{min}$ each day and baseline gait measurements were taken. Two weeks after SCI, the TT and MP + TT groups commenced the training (Fig. 1). The treadmill speed was initially set at $5 \mathrm{~m} / \mathrm{min}$ for $5 \mathrm{mins}$, twice/day, 6 days/week, and then the speed was increased by $1 \mathrm{~m} / \mathrm{min}$ per week for 8 weeks. An excitatory stimulation at $0.05 \mathrm{~mA}$ for $1-2 \mathrm{sec}$ was applied to encourage the rats to continue running for the duration of the training.

Gait analysis. Gait recording and analysis were performed as previously described (25). Briefly, a small animal gait analyzer for hind limb gait video was used (Clever Sys Inc.). Each rat's gait was recorded five times (20 sec each time), and three clear videos were selected for analysis. First, the speed of the running belt was set, so that the animals in the closed transparent tunnel containing the running tape were not running at their own speed. Next, a high-speed color camera was used to evaluate the ventral surface angle between the gait and the running tape. The video file was delivered to the meter connected to the computer, and TreadScan 4.0 system software (Clever Sys Inc.) was used for analysis. The angle of hind limb gait, hind limb swing time, rear foot pitch, stride time, right foot baseline and hind limb maximum lateral deviation were recorded. The recording and analysis were performed using a double-blinded method by two trained individuals. The basic data were recorded 1 day before SCI, and four rats in each group were used to record the gait at 2-8 weeks after SCI. 


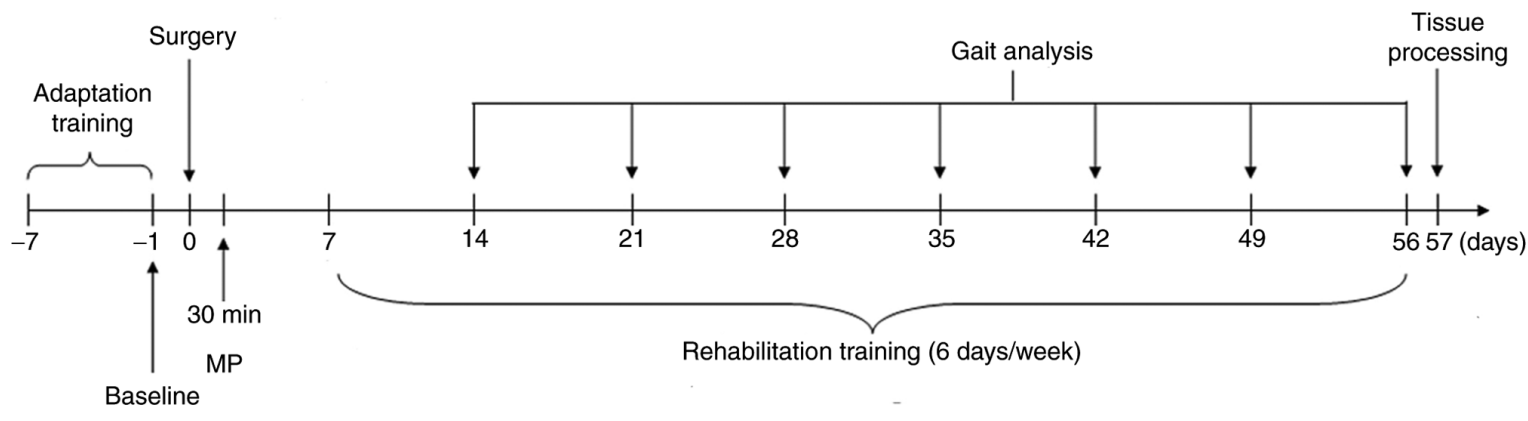

Figure 1. Experimental design timeline. MP, methylprednisolone.

Morphological analysis. A section containing the spinal cord tissue was obtained as previously described (26). Briefly, after completing the behavioral assessment, rats were anesthetized with $5 \%$ chloral hydrate $(300 \mathrm{mg} / \mathrm{kg}$ i.p.; Source Leaf Biological Technology) and subjected to transcardial perfusion with $0.9 \%$ saline followed by $4 \%$ paraformaldehyde (Sigma-Aldrich; Merck KGaA) in 0.1 M PBS (pH 7.4). After perfusion, the spine was removed, and the injured segment was collected (including $\sim 1 \mathrm{~cm}$ of the spinal cord above and below the injury) and placed in $4 \%$ paraformaldehyde solution. The samples were fixed by incubation for 1 day at $4^{\circ} \mathrm{C}$ and dehydrated with a gradient $0.1 \mathrm{~mol} / 1$ cacodylic acid buffer containing 20 and $30 \%$ sucrose. Next, the samples were placed on a HM550 frozen microtome (Microm UK Ltd.), and $20 \mu \mathrm{m}$-thick sections were cut, dried at room temperature and stored at $-80^{\circ} \mathrm{C}$. One slice showing the center of the impact $(+0 \mathrm{~mm})$ and approximately 3 and $5 \mathrm{~mm}$ from the impact was selected and stained with Harris' hematoxylin for $10 \mathrm{~min}$ and with eosin for $1 \mathrm{~min}$ at room temperature. (H\&E; Sigma-Aldrich; Merck KGaA) and Luxol fast blue for $12 \mathrm{~h}$ at $37^{\circ} \mathrm{C}$ (Sigma-Aldrich; Merck KGaA) for routine pathological examination. A total of 10 transversal $20-\mathrm{mm}$ sections from each sample were processed to analyze the cavitation area, and the images were captured by an Olympus DP71 system with an inverted Olympus IX71 microscope (Olympus Corporation; magnification, $\mathrm{x} 40$ ). The myocardium area and the total area of the spinal cord were statistically analyzed with Image-Pro Plus (version 6.0; Media Cybernetics, Inc.). Following completion of sample acquisition, the rats were euthanized by cervical dislocation following anesthesia with intraperitoneal injection of $5 \%$ chloral hydrate $(300 \mathrm{mg} / \mathrm{kg})$.

Immunohistochemistry and image analysis. Immunohistochemistry and image analysis were performed as previously described (27). Four replicate sections of the spinal cord damaged center $(0 \mathrm{~mm})$ and its front and back ( 3 and $5 \mathrm{~mm}$ ) were subjected to immunohistochemistry using the SP-9000 Two-Step Immunohistochemistry kit (OriGene Technologies, Inc.). Briefly, tissue sections were fixed in cold acetone (Sigma-Aldrich; Merck KGaA) at $100 \%$ for $10 \mathrm{~min}$ at $4{ }^{\circ} \mathrm{C}$ and incubated in $3 \%$ deionized aqueous solution for 5-10 min at room temperature to eliminate endogenous peroxidase activity. Next, tissues were washed with $0.01 \mathrm{M}$ PBS three times at 3 min each time. Subsequently, tissues were blocked with $10 \%$ normal goat serum (Beijing Solarbio Science $\&$ Technology Co., Ltd.) in PBS containing 0.2\% Triton X-100 for 10-15 min at room temperature, and then washed with PBS. The sections were then incubated with primary antibodies against NgR (rabbit; 1:10,000; cat. no. AB15138; Merck KGaA) or CSPGs (rabbit; 1:10,000; cat. no. 980704W; Shanghai Tianyuan Biotechnology Co., Ltd.) for $3 \mathrm{~h}$ at room temperature. Subsequently, a biotinylated secondary antibody working fluid $\operatorname{lgG}$ (1:200; cat. no. SP-9000; OriGene Technologies, Inc.) in blocking solution (5\% goat serum in PBS; Beijing Solarbio Science \& Technology Co., Ltd.) was added, and the sections were incubated at $37^{\circ} \mathrm{C}$ for $15 \mathrm{~min}$. Subsequently, the sections were washed with $0.01 \mathrm{M}$ PBS three times (5 min each time), HRP-labeled streptavidin working solution in blocking solution was added and the sections were incubated at $37^{\circ} \mathrm{C}$ for 10-15 min. The sections were then washed again with $0.01 \mathrm{M}$ PBS three times (5 min each time) and 3,3-diaminobenzidine was added to the slices, completely covering the tissue, to develop the color for 5-10 min at room temperature. Next, the tissue sections were placed in hematoxylin solution for 1-2 min for counterstaining at room temperature. The tissues were then subjected to gradient dehydration with $75,80,90,95$ and $100 \%$ ethanol solution, soaked for 2-3 min and submerged in xylene for $\sim 3$ min. Subsequently, 1-2 drops of neutral gum (Bioworld Technology, Inc.) were added to the coverslip for $2 \mathrm{~min}$ at room temperature and covered with a slide. The percentage of positive cells was calculated with Image-Pro Plus software (version 6.0; Media Cybernetics, Inc.) and ImageJ 1.45d image analysis system (National Institutes of Health) by using an inverted Olympus IX71 microscope (Olympus Corporation; magnification, $x 40$ ), as previously described (26). Briefly, the fluoresced cell profiles were counted directly from the screen by placing marks of different colors onto positive and negative nuclei by clicking the mouse. ImageJ then automatically generated the immunohistochemistry index.

CSPGs and $\mathrm{NgR}$ gene expression. To detect CSPG and NgR gene expression, reverse transcription-quantitative PCR (RT-qPCR) was performed as previously described (28). Rats were sacrificed at 8 weeks after the initial injury. Four rats in each group were selected to analyze CSPGs and $\mathrm{NgR}$ gene expression. For this purpose, the spinal cord was collected at $\sim 1 \mathrm{~cm}$ before and after the center of the spinal cord damage. Total RNA extraction and cDNA synthesis were performed as previously described (29). Briefly, total RNA from each individual sample was isolated using the RNeasy Protect Mini kit (Qiagen, Inc.). RNA purity and quantity were assessed using SmartSpec Plus (Bio-Rad Laboratories, Inc.). cDNA 
Table I. Primer sequences used for reverse transcription-quantitative PCR.

\begin{tabular}{llll}
\hline Gene & Primer & Sequence & Product size (bp) \\
\hline $\mathrm{NgR}$ & Forward & 5'-ACCCTCCCATGTAGACGTCTCC-3' & 637 \\
& Reverse & 5'-CTGAAGAGCAATGTTGTTTAGC-3' & 960 \\
CSPG & Forward & 5'-GATGGAATCTTTCACCCAAGAA-3 & 961 \\
& Reverse & 5'-ACCGAGTTGAGCTTTGTCCAAC-3 & \\
& Forward & 5'-GGCACCACACCTTCTACAAT-3' & \\
& Reverse & 5'-TAGCCTTGGCGAGCAACGGT-3' &
\end{tabular}

NgR, Nogo receptor; CSPG, chondroitin sulfate proteoglycan.

synthesis was performed using the iScript cDNA Synthesis kit (Bio-Rad Laboratories, Inc.) in accordance with the manufacturer's instructions. RT-qPCR analysis was performed using the iQ5 instrument (Bio-Rad Laboratories, Inc.) and detected with SYBR Green (BioChain Institute, Inc.). The reaction mixtures contained diluted cDNA, Super Mix (Invitrogen; Thermo Fisher Scientific, Inc.), PCR primers (Table I), and nuclease-free water to a final volume of $20 \mu \mathrm{l}$. The following thermocycling conditions were used for the qPCR: Initial denaturation at $95^{\circ} \mathrm{C}$ for $30 \mathrm{sec}$, followed by 40 cycles of $95^{\circ} \mathrm{C}$ for $15 \mathrm{sec}$ and $60^{\circ} \mathrm{C}$ for $35 \mathrm{sec}$. Relative quantification of gene expression was calculated using the $2^{-\Delta \Delta \mathrm{Cq}}$ method (30). The primers were obtained and synthesized by Invitrogen (Thermo Fisher Scientific, Inc.). $\beta$-actin was used as the internal reference (28).

Statistical analysis. SPSS software 16.0.2 (SPSS, Inc.) was used for statistical analysis. All data were expressed as the mean \pm SD. Comparisons between groups were analyzed using one-way ANOVA followed by Tukey's post hoc test. $\mathrm{P}<0.05$ was considered to indicate a statistically significant difference.

\section{Results}

$M P$ and TT combined therapy improves the recovery of locomotor function. To investigate whether MP and TT combination improved the recovery of motor function in rats with SCI, the locomotor function of each group was compared using the TreadScan analysis system. The motor function of the affected hind limbs was evaluated at the beginning of the 2 -week period post-SCI and weekly thereafter for 8 weeks (Fig. 2). TreadScan analysis revealed that the combined treatment of MP and TT resulted in a significantly higher gait angle compared with the MP and TT groups $(\mathrm{P}<0.01$; Fig. $2 \mathrm{~A})$. At 2-3 weeks post-injury, MP + TT treatment exerted a significant decrease in the distance between the bilateral hind legs and the body's horizontal axis compared with the effects of MP and TT treatment $(\mathrm{P}<0.05)$, and the difference was more significant at 4-8 weeks post-SCI $(\mathrm{P}<0.01$; Fig. $2 \mathrm{~B})$. The time required for the rats under $\mathrm{MP}+\mathrm{TT}$ treatment to perform the walk step test was significantly lower compared with rats under MP and TT treatment at 2-4 weeks post-SCI $(\mathrm{P}<0.05)$, and this difference was more significant at $5-8$ weeks post-injury $(\mathrm{P}<0.01$; Fig. 2C). The hind limb swing time of the rats subjected to $\mathrm{MP}+\mathrm{TT}$ treatment was significantly lower compared with rats under MP and TT treatment at 2-4 weeks post-SCI $(\mathrm{P}<0.01)$, and a significant difference between the two groups was still present at 6-8 weeks post-injury $(\mathrm{P}<0.05$; Fig. 2D). Rats under $\mathrm{MP}+\mathrm{TT}$ treatment at 6-7 weeks post-SCI showed a significant reduction in rear foot pitch compared with rats under MP and TT treatment $(\mathrm{P}<0.01$; Fig. $2 \mathrm{E})$. The distance between the right foot and the baselinein the MP + TT group was significantly smaller than that observed in the MP group at 2-4 weeks after SCI $(\mathrm{P}<0.01)$, and this difference was still significant at 8 weeks after injury $(\mathrm{P}<0.05$; Fig. $2 \mathrm{~F})$. These results suggested that MP + TT treatment was more effective than MP and TT alone in promoting the recovery of hind limb motor function in rats with SCI.

$M P$ and TT combined therapy increases the residual myelin area. To determine the extent of tissue destruction in each group, H\&E and Luxol solid blue staining were used. The results of H\&E staining in Fig. 3 showed that the SCI, TT, $\mathrm{MP}+\mathrm{TT}$ and MP groups had different degrees of tissue loss and cyst appearance at 8 weeks after injury. Although the $\mathrm{MP}+\mathrm{TT}$ group showed fibrous scar formation in the periphery of the lesion, the total glial cell proliferation, tissue gap and cyst area were significantly smaller than those observed in the MP and TT groups.

The results of Luxol solid blue staining in Fig. 4 showed no significant difference in residual myelin area among the groups at $\pm 5 \mathrm{~mm}$ from the injury center at 8 weeks after injury (Fig. 4B and F). At the injury center, the residual myelin area in the MP + TT group $(0.353 \pm 0.033)$ was significantly higher compared with the MP group $(0.221 \pm 0.067)(\mathrm{P}<0.05$; Fig. 4D). In addition, a significant difference in residual myelin area between the MP + TT group and the MP group was observed at $\pm 3 \mathrm{~mm}$ from the injury center $(\mathrm{P}<0.01$; Fig. $4 \mathrm{C}$ and $\mathrm{E})$.

$M P$ and TT combination therapy inhibits the expression of $N g R$ and CSPGs. To investigate whether the combined treatment of MP and TT affected nerve regeneration in rats with SCI, NgR and CSPG gene and protein expression were detected in each group. NgR-positive areas were mainly concentrated around the lesion center. With the increase in distance from the lesion center, the expression was gradually decreased (Fig. S1). $\mathrm{NgR}$ expression in the Sham group was significantly lower compared with the MP and TT groups $(\mathrm{P}<0.01$; Fig. 5B-E). In addition, the positive expression of $\mathrm{NgR}$ in other areas in the SCI group was significantly higher than that displayed by 

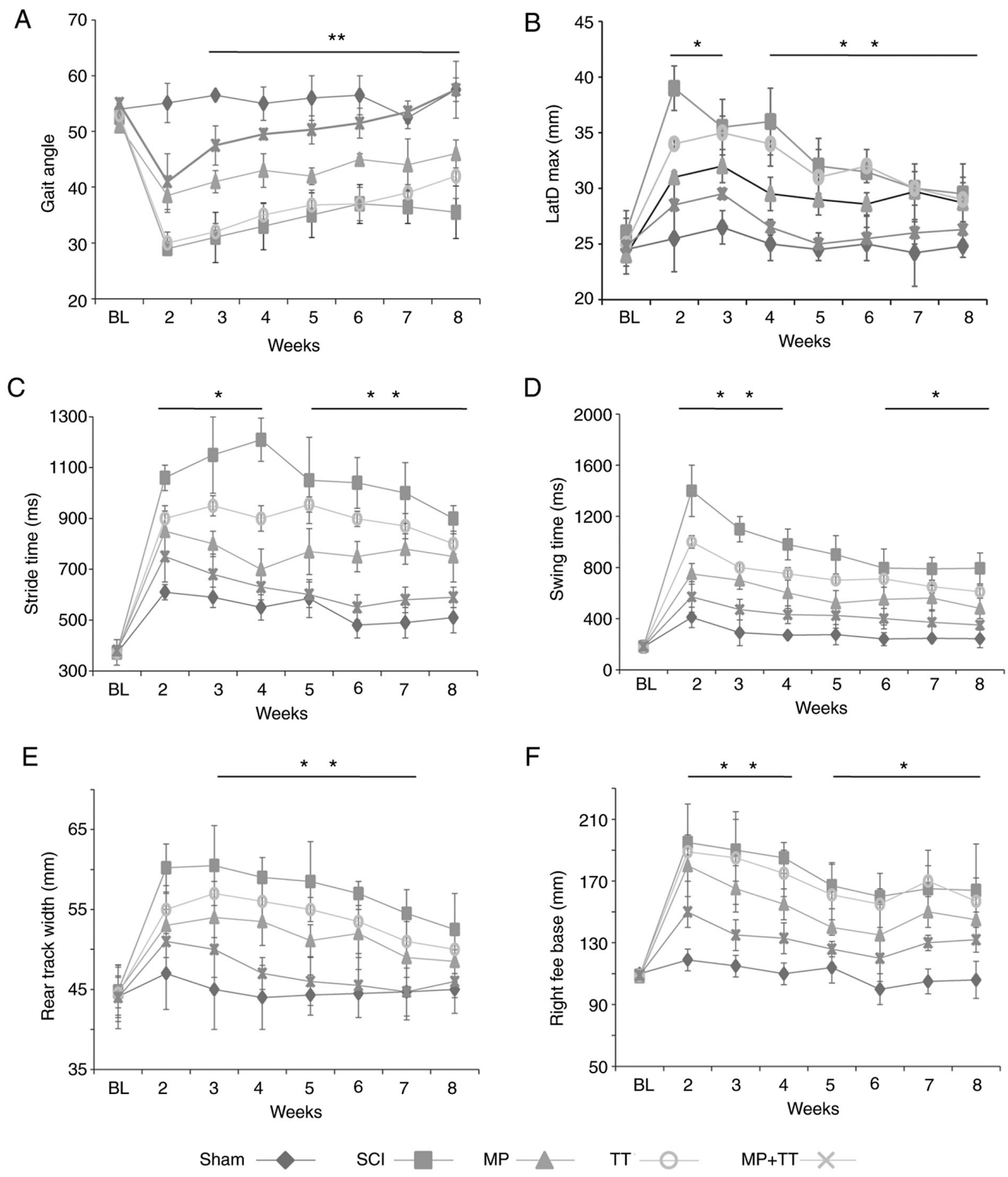

$\mathrm{TT}-\mathrm{MP}+\mathrm{TT}-\mathrm{X}$

Figure 2. Hind limb gait parameters were measured with the TreadScan gait analysis system (before surgery and at 2-8 weeks after injury) in each treatment group. (A) Hind limb gait angle. (B) Maximum lateral deviation. (C) Stride time. (D) Swing time. (E) Rear track width. (F) Right feet base. The results are expressed as the mean $\pm \mathrm{SD}(\mathrm{n}=4)$. Data were analyzed with one-way ANOVA followed by Tukey's post hoctest. " $\mathrm{P}<0.05$ and ${ }^{* *} \mathrm{P}<0.01 \mathrm{vs}$. Sham. LatD Max, maximum lateral deviation; BL, baseline; MP, methylprednisolone; SCI, spinal cord injury; TT, treadmill training.

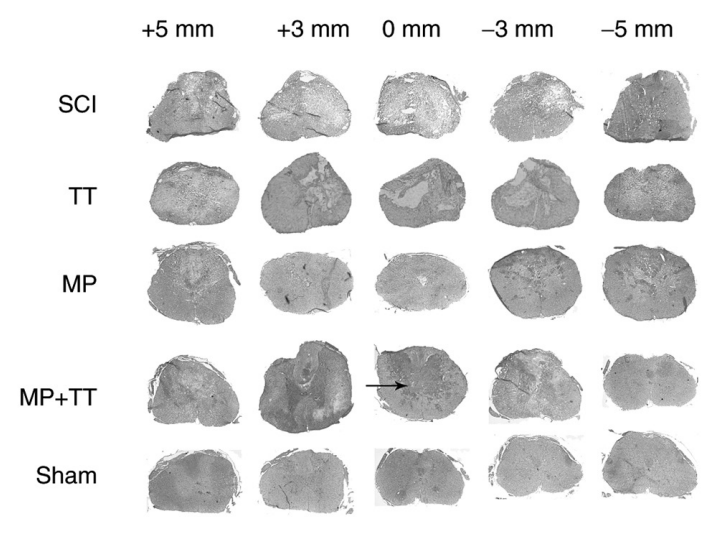

Figure 3. Histopathological changes in the spinal cord following trauma in all groups. Horizontal cross-sections were obtained from the injured site $(+0 \mathrm{~mm})$, and at 3 and $5 \mathrm{~mm}$ rostral (+) and caudal (-) to the epicenter (hematoxylin and eosin staining; magnification, $\mathrm{x} 40$ ). MP, methylprednisolone; SCI, spinal cord injury; TT, treadmill training. the Sham group $(\mathrm{P}<0.01)$. NgR expression in the MP + TT group was significantly lower compared with the MP group at 0 and $\pm 3 \mathrm{~mm}$ from the injury center $(\mathrm{P}<0.01$; Fig. $5 \mathrm{C}-\mathrm{E})$, and the difference was more evident at $\pm 5 \mathrm{~mm}$ from the injury center $(\mathrm{P}<0.01$; Fig. 5B and $\mathrm{F})$.

The results of CSPG immunohistochemical staining are shown in Fig. 6. The positive CSPG areas in each group were mainly concentrated in the center of the injury, spreading to both sides (Fig. S2). CSPG expression in the MP + TT and Sham groups was not significant at $\pm 5 \mathrm{~mm}$ from the injury center compared with the MP group (Fig. 6B and F). At the center of the injury $(0 \mathrm{~mm})$ and at $\pm 3 \mathrm{~mm}$ from this point, CSPG expression in the MP + TT and Sham groups was significantly lower compared with the MP and TT groups $(\mathrm{P}<0.01)$ (Fig. 6C-E).

To further confirm that the MP and TT combination therapy inhibited the expression of $\mathrm{NgR}$ and CSPGs, the mRNA expression of these molecules in the different treatment groups was 
A

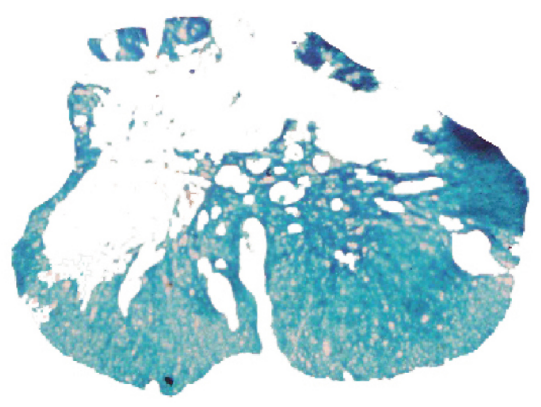

C

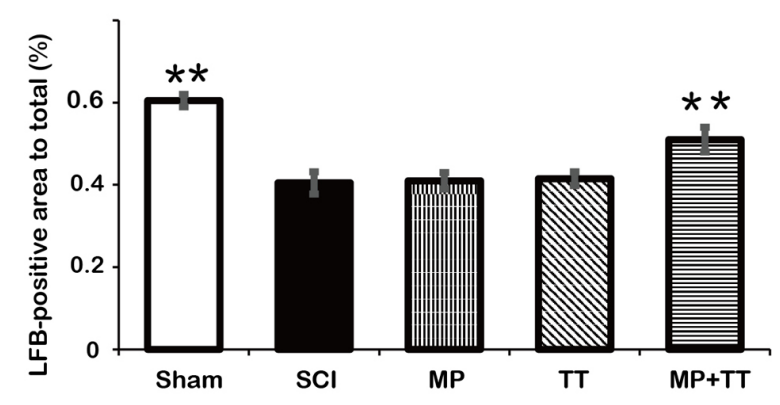

E

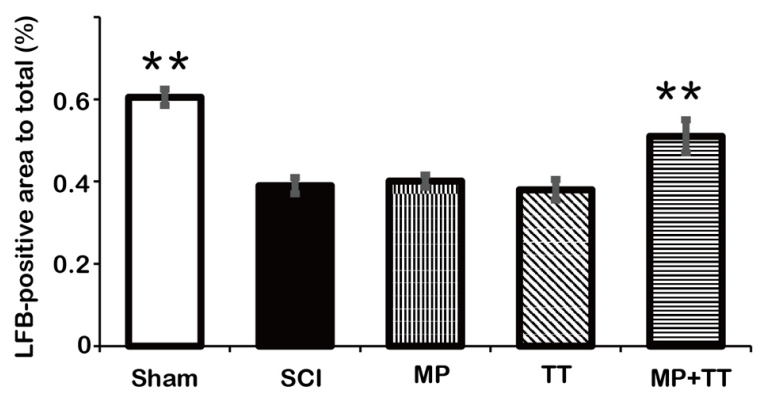

B

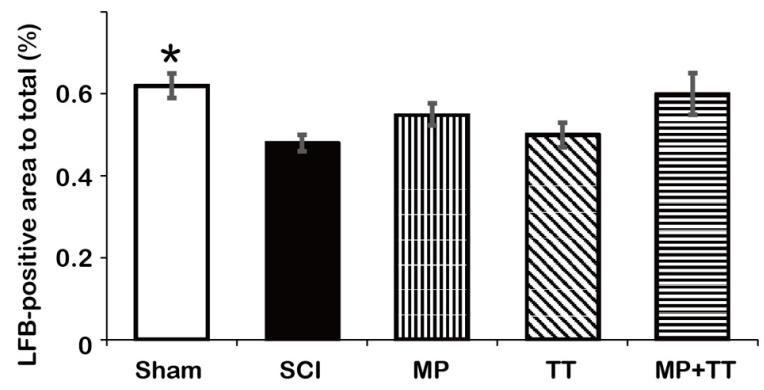

D

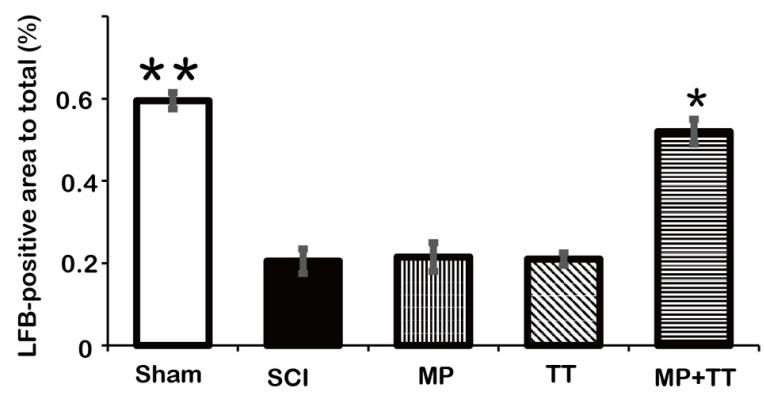

$\mathbf{F}$

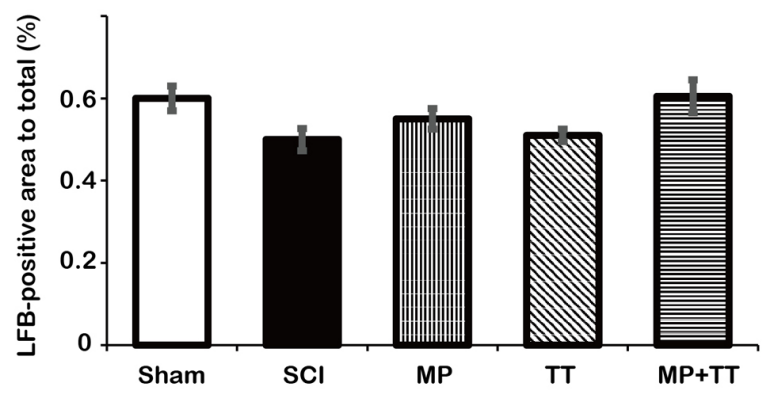

Figure 4. Quantitative comparison of residual myelin areas in all groups at the injured site and at 3 and $5 \mathrm{~mm}$ rostral (+) and caudal (-) from the epicenter after contusive SCI. (A) Statistically analyzed spinal tissue. (B) $+5 \mathrm{~mm},(\mathrm{C})+3 \mathrm{~mm}$, (D) $0 \mathrm{~mm}$, (E) $-3 \mathrm{~mm}$ and (F) $-5 \mathrm{~mm}$. The results are expressed as the mean \pm SD $(\mathrm{n}=4)$. Data were analyzed with one-way ANOVA followed by Tukey's post hoc test. ${ }^{*} \mathrm{P}<0.05$ and ${ }^{* *} \mathrm{P}<0.01$ vs. MP group. MP, methylprednisolone; SCI, spinal cord injury; TT, treadmill training; LFB, Luxol fast blue.

evaluated by fluorescence qPCR. The NgR mRNA expression in the MP + TT group $(0.467 \pm 0.150)$ and in the Sham group $(0.231 \pm 0.102)$ was significantly lower compared with the MP and TT groups $(\mathrm{P}<0.01$; Fig. $7 \mathrm{~A})$. The NgR mRNA expression in the SCI group $(1.413 \pm 0.110)$ was significantly higher compared with the MP and TT groups $(\mathrm{P}<0.05 ;$ Fig. 7A). Similarly, $\mathrm{CSPG}$ mRNA expression in the MP + TT $(0.798 \pm 0.012)$ and Sham $(0.798 \pm 0.012)$ groups was significantly lower compared with the MP group $(\mathrm{P}<0.05$; Fig. 7B). The CSPG mRNA expression in the SCI group $(1.364 \pm 0.259)$ was significantly higher compared with the MP group ( $\mathrm{P}<0.05$; Fig. 7B).

\section{Discussion}

The present study indicated that the combination of MP and treadmill training resulted in cumulative improvements in enhancing the functional recovery of rats after SCI. In addition, significantly reduced $\mathrm{NgR}$ and CSPG protein and mRNA expression were observed. To the best of our knowledge, these findings are the first showing the effect of combined MP and treadmill training treatment following SCI. The present study also demonstrated that the restorative effects of this combination therapy may be mediated by improved nerve regeneration.

The steps in SCI treatment aim to reduce scar formation, slow down the apoptosis of the remaining neurons and strengthen limb activities (31). Therefore, it is necessary to combine MP with other treatment methods to improve post-SCI treatment. Exercise training is one of the effective interventions in the treatment of SCI $(32,33)$, as it reinforces the effects of specific sensory motor pathways, thus resulting in more selective and stable neural network loops (33). Gonzenbach et al (34) found that Nogo-A antibody treatment in combination with exercise therapy can reduce muscle spasm after injury and is beneficial to the recovery of motor function. Treadmill training is one of the most common types of exercise training to enhance SCI recovery (35). It not only uses the hind legs to promote the recovery of animals (23), but can also prevent or restore the loss of synapses, thus enhancing the plasticity of the spinal cord in rats with SCI and resulting in improved lower limb motor 
A

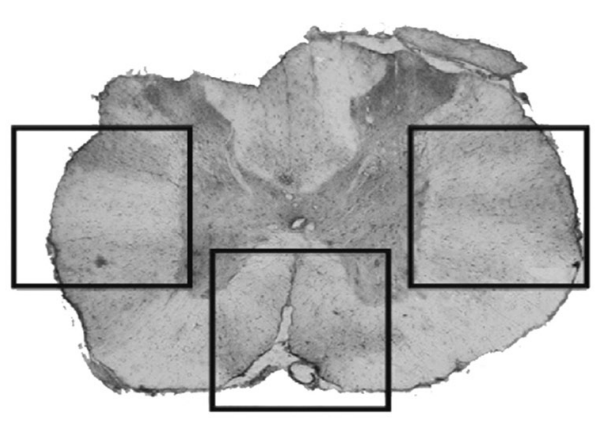

C

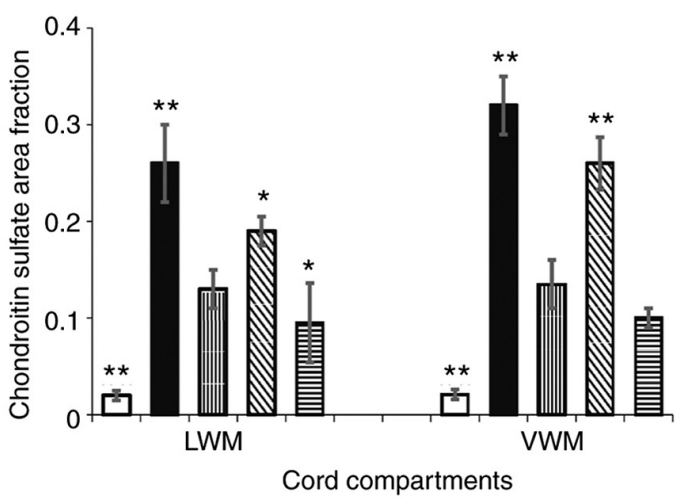

E

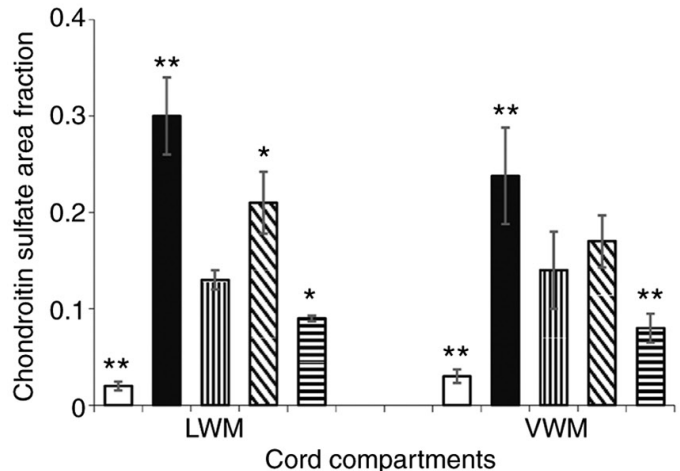

B

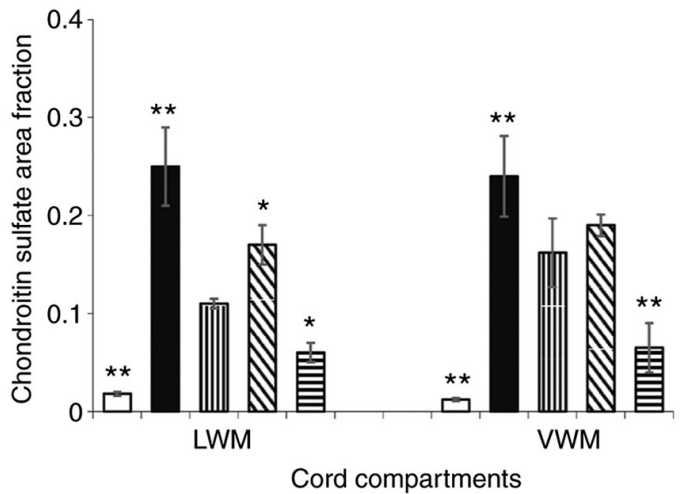

D

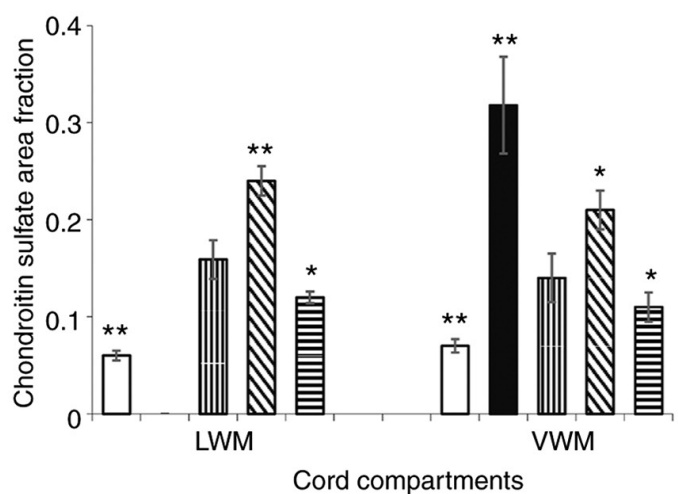

$\mathrm{F}$

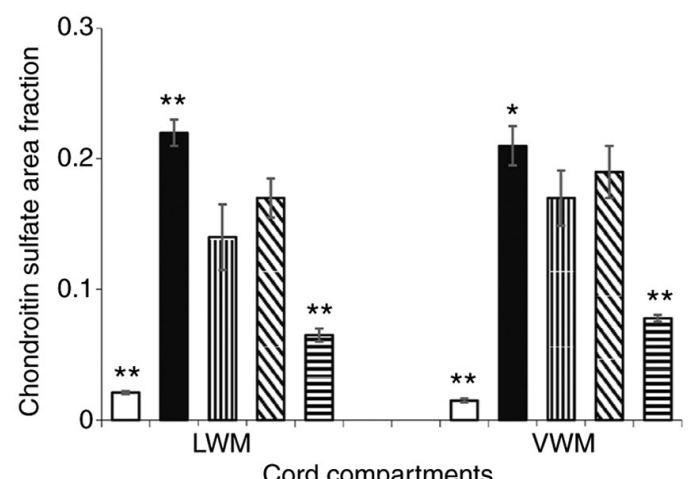

Cord compartments

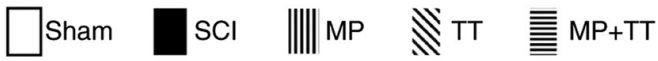

Figure 5. NgR protein expression in specific cord compartments in all groups at the injured site and at 3 and $5 \mathrm{~mm}$ rostral (+) and caudal (-) from the epicenter after contusive SCI. (A) Statistically analyzed spinal tissue. (B) $+5 \mathrm{~mm},(\mathrm{C})+3 \mathrm{~mm}$, (D) $0 \mathrm{~mm}$, (E) $-3 \mathrm{~mm}$ and (F) $-5 \mathrm{~mm}$. The results are expressed as the mean \pm SD $(n=4)$. Data were analyzed with one-way ANOVA followed by Tukey's post hoc test. $\mathrm{P}<0.05$ and ${ }^{* *} \mathrm{P}<0.01$ vs. MP group. NgR, Nogo receptor; LWM, lateral white matter; VWM, ventral white matter; MP, methylprednisolone; SCI, spinal cord injury; TT, treadmill training.

function, which is a significant therapeutic effect $(36,37)$. The results of the present study also demonstrated that the hind limb swing times and rear track width of the rats in the SCI group were significantly higher than those of the rats in the Sham group, which is in accordance with previous reports $(38,39)$. The significantly shorter hind limb swing times, shorter maximum lateral deviation, shorter distance between the right foot and the baseline and narrower rear track width in the MP + TT group indicated hind limb injury recovery, overall movement coordination, hind limb movement and balance ability due to the combined treatment administered to rats with SCI, which exerted a better effect than MP treatment alone.

Glial scar formation is one of the key reasons leading to SCI secondary tissue injury (20). Although the early postoperative scar has a certain positive protective effect (21), it can eventually seriously affect the regeneration of axons (40). Following SCI, astrocytes repair the wound around the damaged tissue, but this also interferes with axonal regrowth (41). Astrocyte-reactive proliferation gradually produces inflammation and fibrosis bundle overlap with the increase in disease time, leading to dislocation of chondroitin sulfate protein deposition, and ultimately promoting glial scar formation (42). $\mathrm{NgR}$ and CSPGs are important factors for inhibiting axonal growth. $\mathrm{NgR}$ directly inhibits axonal regeneration and signal transduction during the germination process in the injured region (18). Previous studies found that inhibition of CSPGs expression effectively improves the microenvironment around SCI, and enhances the expression of MBP and GAP-43 $(20,21,43)$, but still needs to be combined with other treatments to produce 
A

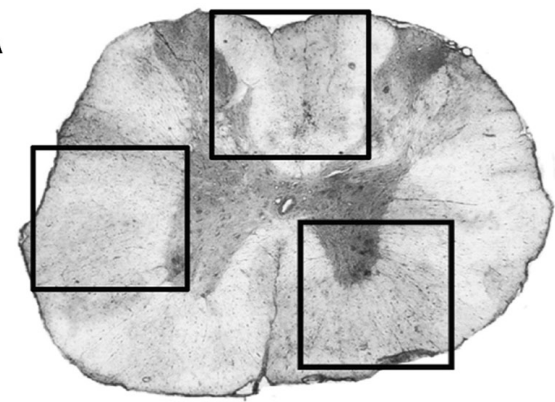

C

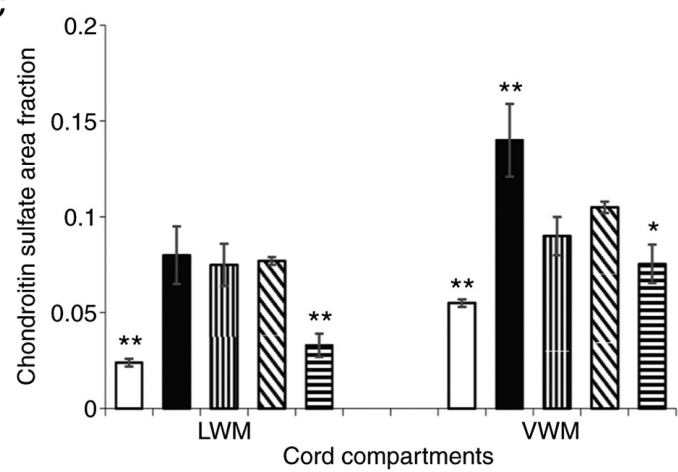

E

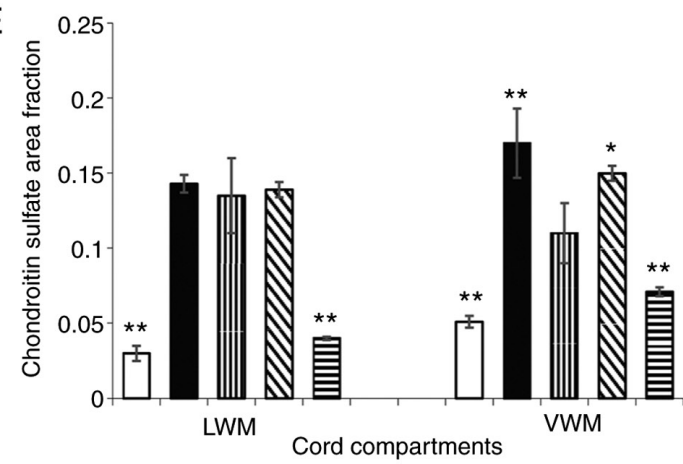

$\mathrm{B}$

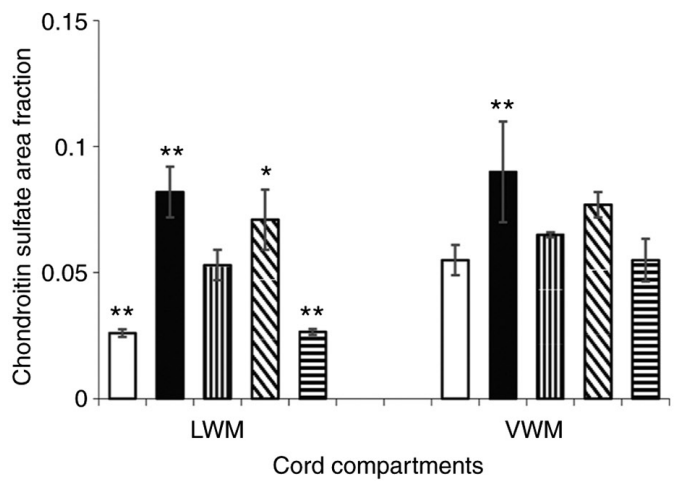

D

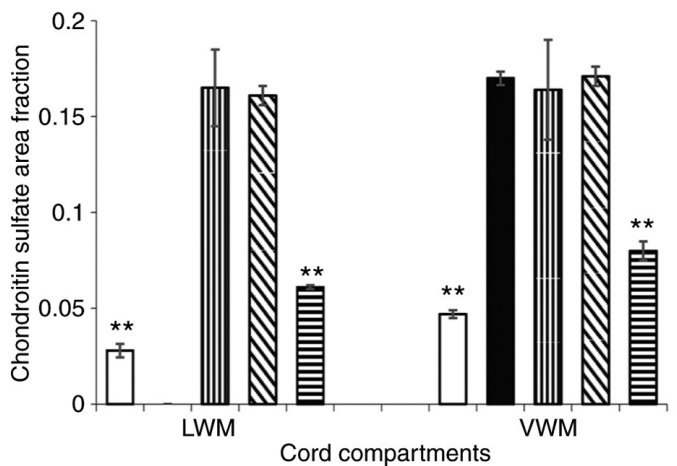

$\mathrm{F}$

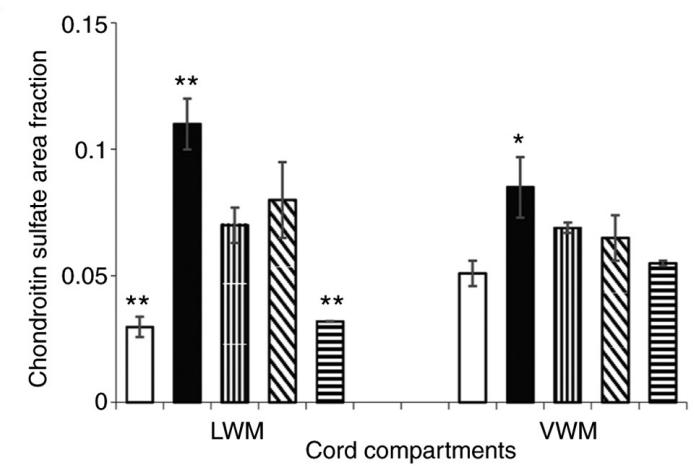

\|\|||$M P \quad$ 热TT

Figure 6. CSPG protein expression in specific cord compartments in all groups at the injured site and at 3 and $5 \mathrm{~mm}$ rostral (+) and caudal (-) from the epicenter after contusive SCI. (A) Statistically analyzed spinal tissue. (B) $+5 \mathrm{~mm}$, (C) $+3 \mathrm{~mm}$, (D) $0 \mathrm{~mm}$, (E) $-3 \mathrm{~mm}$ and (F) $-5 \mathrm{~mm}$. The results are expressed as the mean $\pm \mathrm{SD}(\mathrm{n}=4)$. One-way ANOVA followed by Dunnett's post hoc test was employed for data analysis. ${ }^{*} \mathrm{P}<0.05$ and ${ }^{* * *} \mathrm{P}<0.01$ vs. MP group. CSPGs, chondroitin sulfate proteoglycans; LWM, lateral white matter; VWM, ventral white matter; MP, methylprednisolone; SCI, spinal cord injury; TT, treadmill training.

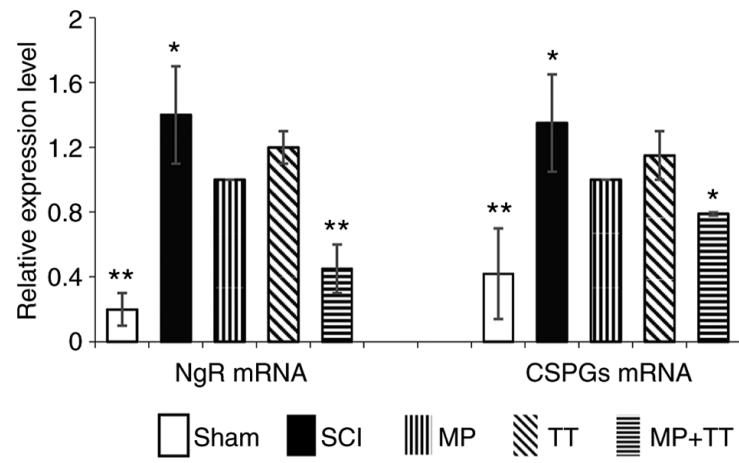

Figure 7. NgR and CSPG mRNA expression was determined by reverse transcription-quantitative PCR in all groups. The results are expressed as the mean $\pm \operatorname{SD}(n=4)$. Data were analyzed with one-way ANOVA followed by Tukey's post hoc test. ${ }^{*} \mathrm{P}<0.05$ and ${ }^{* *} \mathrm{P}<0.01$ vs. MP group. MP, methylprednisolone; SCI, spinal cord injury; TT, treadmill training; NgR, Nogo receptor; CSPGs, chondroitin sulfate proteoglycans. significant axonal regeneration and myelination, reduction in glial scar formation and recovery of nerve function (44). In accordance with previous reports $(18,20,21)$, the present results showed that CSPG and $\mathrm{NgR}$ expression in the SCI group was higher than that in other groups. The expression of CSPGs and $\mathrm{NgR}$ in rats with SCI after combined therapy was significantly lower compared with rats after MP treatment alone. Moreover, by observing the extent of the damage around the malacic spinal cord in this rat model of spinal trauma, the present study similarly found that combined therapy had a certain effect on the reduction of glial scar formation in rats with SCI. Compared with the single MP treatment group, the TT + MP treatment group showed decreased tissue loss and glial scar formation, and demyelination was significantly improved within rostral and caudal $3 \mathrm{~mm}$ from the epicenter. These results suggested that combination therapy might have a 
certain effect on the reduction of glial scar formation and the promotion of nerve repair in rats with SCI.

In conclusion, the present results demonstrated that $\mathrm{MP}+\mathrm{TT}$ treatment is more effective in repairing the motor function of hind limbs in rats with SCI compared with MP treatment alone. Combined therapy inhibited the expression of NgR and CSPGs in SCI, suggesting that it might promote the recovery of nerve function in rats with SCI by reducing the formation of glial scarring. However, the present study also has several limitations. First, the present study mainly focused on the recovery of motor function in SCI rats; the combination therapy on other adverse effects remains to be studied. Second, there are numerous types of cytokines involved in the development of the SCI, such as brain-derived neurotrophic factor and neurotrophin-3, which should be investigated. These experiments and analyses will be performed in future studies, with an emphasis on better understanding the detailed molecular mechanisms of combination therapy restoration function in SCI rats.

\section{Acknowledgements}

Not applicable.

\section{Funding}

The present study was supported by the National Natural Science Foundation of China (grant no. 30960448); the Natural Science Foundation of Jiangxi Province (grant no. 20142BAB205023) and the China Scholarship Council (grant no. 201908360006).

\section{Availability of data and materials}

The datasets used and/or analyzed during the current study are available from the corresponding author on reasonable request.

\section{Authors' contributions}

BG designed the study. SW and HX performed the experiments. JL and HL analyzed the data. JL and BG wrote the manuscript. BG and JL confirm the authenticity of all the raw data All authors read and approved the final manuscript.

\section{Ethics approval and consent to participate}

The study protocol was approved by the Animal Ethics Committee of Jiangxi University (approval no. A20150107). The experimental procedure followed the United States National Institutes of Health Guide for the Care and Use of Laboratory Animals (NIH publication no. 85-23, revised 1986).

\section{Patient consent for publication}

Not applicable.

\section{Competing interests}

The authors declare that they have no competing interests.

\section{References}

1. Selfslagh A, Shokur S, Campos DSF, Donati ARC, Almeida S, Yamauti SY, Coelho DB, Bouri M and Nicolelis MAL: Non-invasive, brain-controlled functional electrical stimulation for locomotion rehabilitation in individuals with paraplegia. Sci Rep 9: 6782, 2019.

2. Silvestro S, Bramanti P, Trubiani O and Mazzon E: Stem cells therapy for spinal cord injury: An overview of clinical trials. Int J Mol Sci 21: 659, 2020.

3. Chen XG, Chen LH, Xu RX and Zhang HT: Effect evaluation of methylprednisolone plus mitochondrial division inhibitor- 1 on spinal cord injury rats. Childs Nerv Syst 34: 1479-1487, 2018.

4. Ahuja CS, Wilson JR, Nori S, Kotter MRN, Druschel C, Curt A and Fehlings MG: Traumatic spinal cord injury. Nat Rev Dis Primers 3: 17018, 2017.

5. Bowers CA, Kundu B and Hawryluk GW: Methylprednisolone for acute spinal cord injury: An increasingly philosophical debate. Neural Regen Res 11: 882-885, 2016.

6. Park SH and Cho KH: Large-dose glucocorticoid induced secondary adrenal insufficiency in spinal cord injury. Ann Rehabil Med 40: 1033-1039, 2016.

7. Sunshine JE, Dagal A, Burns SP, Bransford RJ, Zhang F, Newman SF, Nair BG and Sharar SR: Methylprednisolone therapy in acute traumatic spinal cord injury: Analysis of a regional spinal cord model systems database. Anesth Analg 124: 1200-1205, 2017.

8. Li XG, Lin XJ, Du JH, Xu SZ, Lou XF and Chen Z: Combination of methylprednisolone and rosiglitazone promotes recovery of neurological function after spinal cord injury. Neural Regen Res 11: 1678-1684, 2016.

9. Mbori NJ, Chuan XY,Feng QX, Alizada M and Zhan J: Evaluation of the combination of methylprednisolone and tranilast after spinal cord injury in rat models. J Korean Neurosurg Soc 59: 334-340, 2016.

10. Yao NW, Lu Y, Shi LQ, Xu F and Cai XH: Neuroprotective effect of combining tanshinone IIA with low-dose methylprednisolone following acute spinal cord injury in rats. Exp Ther Med 13: 2193-2202, 2017.

11. Karsy M and Hawryluk G: Pharmacologic management of acute spinal cord injury. Neurosurg Clin N Am 28: 49-62, 2017.

12. Chang YX, Zhao Y, Pan S, Qi ZP, Kong WJ, Pan YR, Li HR and Yang XY: Intramuscular injection of adenoassociated virus encoding human neurotrophic factor 3 and exercise intervention contribute to reduce spasms after spinal cord injury. Neural Plast 2019: 3017678, 2019.

13. Chen K, Marsh BC, Cowan M, Al'Joboori YD, Gigout S, Smith CC, Messenger N, Gamper N, Schwab ME and Ichiyama RM: Sequential therapy of anti-Nogo-A antibody treatment and treadmill training leads to cumulative improvements after spinal cord injury in rats. Exp Neurol 292: $135-144,2017$.

14. Harnie J, Doelman A, de Vette E, Audet J, Desrochers E, Gaudreault $\mathrm{N}$ and Frigon A: The recovery of standing and locomotion after spinal cord injury does not require task-specific training. Elife 8: e50134, 2019.

15. Martinez SA, Nguyen ND, Bailey E, Doyle-Green D, Hauser HA, Handrakis JP, Knezevic S, Marett C, Weinman J, Romero AF, et al: Multimodal cortical and subcortical exercise compared with treadmill training for spinal cord injury. PLoS One 13: e0202130, 2018.

16. Younsi A, Zheng G, Scherer M, Riemann L, Zhang H, Tail M, Hatami M, Skutella T, Unterberg A and Zweckberger K: Treadmill training improves survival and differentiation of transplanted neural precursor cells after cervical spinal cord injury. Stem Cell Res 45: 101812, 2020.

17. Yao Y, Xu J, Yu T, Chen Z, Xiao Z, Wang J, Hu Y, Wu Y and Zhu D: Flufenamic acid inhibits secondary hemorrhage and BSCB disruption after spinal cord injury. Theranostics 8: 4181-4198, 2018

18. Sekine Y, Siegel CS, Sekine-Konno T, Cafferty WBJ and Strittmatter SM: The nociceptin receptor inhibits axonal regeneration and recovery from spinal cord injury. Sci Signal 11: eaao4180, 2018.

19. Hirokawa $\mathrm{T}$ and Takei K: Lateral olfactory tract usher substance (LOTUS) protein, an endogenous Nogo receptor antagonist, converts a non-permissive to permissive brain environment for axonal regrowth. Neural Regen Res 13: 1193-1194, 2018. 
20. Wu D, Jin Y, Shapiro TM, Hinduja A, Baas PW and Tom VJ: Chronic neuronal activation increases dynamic microtubules to enhance functional axon regeneration after dorsal root crush injury. Nat Commun 11: 6131, 2020.

21. Xu B, Park D, Ohtake Y, Li H, Hayat U, Liu J, Selzer ME, Longo FM and Li S: Role of CSPG receptor LAR phosphatase in restricting axon regeneration after CNS injury. Neurobiol Dis 73: $36-48,2015$

22. Wu D, Klaw MC, Connors T, Kholodilov N, Burke RE and Tom VJ: Expressing constitutively active Rheb in adult neurons after a complete spinal cord injury enhances axonal regeneration beyond a chondroitinase-treated glial scar. J Neurosci 35: 11068-11080, 2015.

23. Battistuzzo CR, Rank MM, Flynn JR, Morgan DL, Callister R, Callister RJ and Galea MP: Effects of treadmill training on hindlimb muscles of spinal cord-injured mice. Muscle Nerve 55: 232-242, 2017.

24. National Research Council (US) Institute for Laboratory Animal Research: Guide for the care and use of laboratory animals. National Academies Press, Washington (DC), 1996.

25. Gu B, Jin JB and Li HN: Establishment of traumatic spinal cord injury model in rats. Chin J Clin Pharmacol Ther 16: 8, 2011 (In Chinese)

26. Zhang S, Gu B, Wang SY, Li HN, Zhang GF and Zhang SY: Application of treadscan gait analysis system in the evaluation of rat spinal cord contusion model. Acta Neuropharmacologica 2: 3 , 2012 (In Chinese).

27. Zong H, Li X, Lin H, Hou C and Ma F: Lipoxin A4 pretreatment mitigates skeletal muscle ischemia-reperfusion injury in rats. Am J Transl Res 9: 1139-1150, 2017.

28. Yang G, Yang Y, Tang H and Yang K: Loss of the clock gene Perl promotes oral squamous cell carcinoma progression via the AKT/mTOR pathway. Cancer Sci 111: 1542-1554, 2020.

29. Liu JT, Zhang S, Gu B, Li HN, Wang SY and Zhang SY: Methotrexate combined with methylprednisolone for the recovery of motor function and differential gene expression in rats with spinal cord injury. Neural Regen Res 12: 1507-1518, 2017.

30. Liu J, Zhu L, Zhang S, Deng Z, Huang Z, Yuan M, $\mathrm{Wu} \mathrm{W}$ and Yang $\mathrm{K}$ : The autographa californica multiple nucleopolyhedrovirus ac110 gene encodes a new per os infectivity factor. Virus Res 221: 30-37, 2016.

31. Fischer I, Dulin JN and Lane MA: Transplanting neural progenitor cells to restore connectivity after spinal cord injury. Nat Rev Neurosci 21: 366-383, 2020.

32. Li J, Polston KFL, Eraslan M, Bickel CS, Windham ST, McLain AB, Oster RA, Bamman MM and Yarar-Fisher C: A high-protein diet or combination exercise training to improve metabolic health in individuals with long-standing spinal cord injury: A pilot randomized study. Physiol Rep 6: e13813, 2018.
33. West CR, Campbell IG, Goosey-Tolfrey VL, Mason BS and Romer LM: Effects of abdominal binding on field-based exercise responses in paralympic athletes with cervical spinal cord injury. J Sci Med Sport 17: 351-355, 2014.

34. Gonzenbach RR, Gasser P, Zorner B, Hochreutener E, Dietz V and Schwab ME: Nogo-A antibodies and training reduce muscle spasms in spinal cord-injured rats. Ann Neurol 68: 48-57, 2010.

35. Kim HN, Pak ME, Shin MJ, Kim SY, Shin YB, Yun YJ, Shin HK and Choi BT: Comparative analysis of the beneficial effects of treadmill training and electroacupuncture in a rat model of neonatal hypoxia-ischemia. Int J Mol Med 39: 1393-1402, 2017.

36. Heng $C$ and de Leon RD: Treadmill training enhances the recovery of normal stepping patterns in spinal cord contused rats. Exp Neurol 216: 139-147, 2009.

37. Wang H, Liu NK, Zhang YP, Deng L, Lu QB, Shields CB, Walker MJ, Li J and Xu XM: Treadmill training induced lumbar motoneuron dendritic plasticity and behavior recovery in adult rats after a thoracic contusive spinal cord injury. Exp Neurol 271: 368-378, 2015

38. Beare JE, Morehouse JR, DeVries WH, Enzmann GU, Burke DA, Magnuson DS and Whittemore SR: Gait analysis in normal and spinal contused mice using the TreadScan system. J Neurotrauma 26: 2045-2056, 2009.

39. McMackin MZ, Henderson CK and Cortopassi GA: Neurobehavioral deficits in the KIKO mouse model of Friedreich's ataxia. Behav Brain Res 316: 183-188, 2017.

40. Wang XJ, Peng CH, Zhang S, Xu XL, Shu GF, Qi J, Zhu YF, Xu DM, Kang XQ, Lu KJ, et al: Polysialic-acid-based micelles promote neural regeneration in spinal cord injury therapy. Nano Lett 19: 829-838, 2019.

41. Yao S, Yu S, Cao Z, Yang Y, Yu X, Mao HQ, Wang LN, Sun X, Zhao $L$ and Wang $X$ : Hierarchically aligned fibrin nanofiber hydrogel accelerated axonal regrowth and locomotor function recovery in rat spinal cord injury. Int J Nanomedicine 13: 2883-2895, 2018

42. Goulão M, Ghosh B, Urban MW, Sahu M, Mercogliano C, Charsar BA, Komaravolu S, Block CG, Smith GM, Wright MC and Lepore AC: Astrocyte progenitor transplantation promotes regeneration of bulbospinal respiratory axons, recovery of diaphragm function, and a reduced macrophage response following cervical spinal cord injury. Glia 67: 452-466, 2019.

43. Bradbury EJ and Carter LM: Manipulating the glial scar: Chondroitinase $\mathrm{ABC}$ as a therapy for spinal cord injury. Brain Res Bull 84: 306-316, 2011.

44. Mukherjee N, Nandi S, Garg S, Ghosh S, Ghosh S, Samat R and Ghosh S: Targeting chondroitin sulfate proteoglycans: An emerging therapeutic strategy to treat CNS injury. ACS Chem Neurosci 11: 231-232, 2020 